\title{
Amikacin-resistant Acinetobacter species mediated by the aphA6 gene associated with clinical outcome at an academic complex hospital in KwaZulu-Natal Province, South Africa
}

\author{
K Swe Swe-Han, ${ }^{1,2}$ MBBS, DTM\&H, PDIC, FC Path (SA) (Medical Microbiology), MMed (Medical Microbiology), \\ $\mathrm{PhD}$ (Medical Microbiology); M Pillay, ${ }^{2,3}$ BMedSc, BMedSc Hons (Microbiology), MSc (Pharmaceutical Chemistry); \\ M Pillay, ${ }^{1}$ BSc, BSc Hons, MMedSc (Medical Microbiology), PhD (Medical Microbiology)
}

\author{
${ }^{1}$ Department of Medical Microbiology, School of Laboratory Medicine and Medical Sciences, College of Health Sciences, University of KwaZulu- \\ Natal, Durban, South Africa \\ ${ }^{2}$ National Health Laboratory Service, Durban, South Africa \\ ${ }^{3}$ Department of Virology, School of Laboratory Medicine and Medical Sciences, College of Health Sciences, University of KwaZulu-Natal, Durban, \\ South Africa
}

Corresponding author: K Swe Swe-Han (sweswe-han@ukzn.ac.za)

\begin{abstract}
Background. Drug-resistant Acinetobacter species present serious therapeutic and infection control policy challenges globally. Although aminoglycosides have played a crucial role in the treatment of infections with multidrug-resistant (MDR) Acinetobacter spp., recent reports indicate that these bacteria are developing resistance to aminoglycosides around the globe.

Objectives. To determine the association between amikacin resistance and clinical outcomes of patients. The minimum inhibitory concentrations (MICs) of amikacin against Acinetobacter spp. and genes associated with resistance were also investigated.

Methods. Clinical information from 107 patients with Acinetobacter spp. cultured from clinical specimens was recorded during ward rounds at an academic complex hospital in KwaZulu-Natal Province, South Africa, including clinical outcomes, history of antibiotics prescribed and microbiological investigations. The 107 Acinetobacter isolates were investigated for susceptibility to antimicrobial agents in use at local hospitals. Genes related to amikacin resistance ( $a p h \mathrm{~A} 6$ and $a a c \mathrm{~A} 4)$ were investigated by polymerase chain reaction (PCR) and sequencing. Analysis was performed on the relationship between clinical outcomes and antimicrobial resistance patterns, as well as on the amikacin MICs in resistant isolates $(n=6) \mathrm{v}$. their PCR results.

Results. The majority $(5 / 6,83.3 \%)$ of patients with amikacin-resistant Acinetobacter infection were discharged, and 1/6 (16.7\%) died. No underlying clinical factors were significantly associated with clinical outcome. Amikacin resistance was observed in 6/107 isolates (5.6\%), with MICs of $32 \mu \mathrm{g} / \mathrm{mL}(n=3)$ and $\geq 64 \mu \mathrm{g} / \mathrm{mL}(n=3)$ for the amikacin-resistant isolates. All 6 of these isolates were also extensively drugresistant (XDR). The aphA6 gene (797 base pair) was detected in all amikacin-resistant isolates.

Conclusions. Most tested Acinetobacter isolates were susceptible to amikacin, underscoring the crucial role of this antibiotic in the treatment of MDR Acinetobacter spp. in our hospital. The emergence of XDR isolates is of serious concern and necessitates close monitoring and surveillance.
\end{abstract}

S Afr Med J 2020;110(1):49-54. https://doi.org/10.7196/SAMJ.2020.v110i1.14045

Acinetobacter species have emerged as major hospital-associated pathogens, which have evolved into multidrug-resistant (MDR) and extensively drug-resistant (XDR) strains during the past decade. ${ }^{[1]}$ Acinetobacter spp. have the capacity to acquire resistance to antimicrobial agents through genetic factors such as plasmids and pathogenicity islands ${ }^{[2]}$ resulting in resistant strains that are difficult to treat. ${ }^{[3]}$ The Infectious Diseases Society of America has therefore declared Acinetobacter spp. among the six antimicrobial-resistant pathogens responsible for high morbidity and mortality. ${ }^{[3,4]}$

Although Acinetobacter spp. are common colonisers that may lead to community-acquired infection, they are also opportunistic pathogens often found in immunocompromised patients with prolonged hospitalisation. ${ }^{[5]}$ Immunosuppressive therapy places cancer patients at risk of developing Acinetobacter infections that may result in sepsis, respiratory infections, wound infections and urinary tract infections. ${ }^{[3,6-8]}$
XDR Acinetobacter spp. are defined as being resistant to all the tested antimicrobials except colistin, whereas pandrug-resistant (PDR) isolates are resistant to all agents. ${ }^{[9]} \mathrm{A}$ rise in infections from XDR Acinetobacter spp. has been reported. ${ }^{[10,11]}$ The global rise of MDR Acinetobacter spp. and the emergence of XDR Acinetobacter spp. therefore pose a major challenge to current treatment options and infection control. ${ }^{[12,13]}$

Until recently, amikacin was the most active aminoglycoside in the treatment of infections caused by Acinetobacter spp. in academic complex hospitals in KwaZulu-Natal Province, South Africa (SA). It remains the drug of choice for treatment of MDR Acinetobacter infections, yet resistance has increased in recent years. ${ }^{[14]}$

Acinetobacter spp. have several mechanisms of aminoglycoside resistance. ${ }^{[15,16]}$ In general, the major mechanism in Gram-negative bacteria is enzymatic modification of the amino or hydrolol groups of the agent through aminoglycoside-modifying enzymes. 
Amikacin is commonly used at Inkosi Albert Luthuli Central Hospital (IALCH), an academic complex hospital in Durban, KwaZulu-Natal, owing to the increasing prevalence of MDR Acinetobacter spp., especially for nebulisation of pneumonia and in combination with piperacillin-tazobactam for systemic infections.

\section{Objectives}

To characterise Acinetobacter spp. isolates and compare the clinical outcomes of infected patients with the phenotypic and genotypic characteristics of XDR Acinetobacter spp. at IALCH.

\section{Methods}

The study received ethical approval from the Biomedical Research Ethics Committee (BREC), College of Health Sciences, University of KwaZulu-Natal (ref. no. BE 283/12).

\section{Study design}

The study was analytical and observational experimental research that highlighted the prevalence of amikacin-resistant Acinetobacter spp., clinical outcomes, and association with genes aphA6 and aacA4, related to amikacin resistance.

\section{Patients and bacterial isolates}

Clinical information on 107 patients with Acinetobacter spp. cultured from clinical specimens was recorded during clinical ward rounds at IALCH. The information included clinical outcomes, history of antibiotics prescribed at local hospitals as part of routine management, and antimicrobial susceptibility patterns of the 107 Acinetobacter isolates.

The minimum inhibitory concentrations (MICs) for 60 of the 107 Acinetobacter isolates were investigated. Six amikacin-resistant clinical isolates of Acinetobacter spp. were selected for genotypic characterisation at the Microbiology Laboratory, National Health Laboratory Service, Durban.

\section{Susceptibility testing}

Susceptibility testing was performed using the VITEK 2 automated system (BioMérieux, France) with the VITEK 2 GN ID card and the VITEK 2 AST-N255 card. The MICs of the appropriate antimicrobial agents in use were determined for 60 Acinetobacter isolates using the Epsilometer test (E-test) (BioMérieux, France). The $\mathrm{MIC}_{90}$ and $\mathrm{MIC}_{50}$ were determined for each antibiotic agent tested against the 60 isolates. The antibiotics included amikacin, carbapenems (imipenem, meropenem), ceftazidime, ciprofloxacin, colistin and piperacillin-tazobactam. Acinetobacter ATCC 19606 was used as the quality control strain. The results were interpreted according to the Clinical and Laboratory Standards Institute. ${ }^{[17]}$ An MIC $>32 \mu \mathrm{g} / \mathrm{mL}$ for amikacin was considered to indicate resistance. ${ }^{[17]}$

\section{Polymerase chain reaction and sequencing}

Genomic DNA from each of 13 isolates, comprising 6 clinically amikacin-resistant strains, 3 controls and 4 known sensitive clinical isolates, was extracted using a previously described method. ${ }^{[18]}$

The presence of the genes related to amikacin resistance (aph $\mathrm{A} 6$ and aacA4) was further investigated by polymerase chain reaction (PCR). The MICs of amikacin ( $n=6)$ were compared with the PCR results of these resistant isolates and clinical outcome.

\section{Clinical and laboratory data collection}

Clinical and laboratory data on 107 patients are reported here. The data included demographics, underlying medical condition, type of specimen, exposure to antimicrobial agents before and after isolation of Acinetobacter spp., admission to intensive care units (ICUs) or other units, and clinical outcomes. The clinicians defined the type of infection. Patients who did not receive specific treatment for Acinetobacter spp. were classified as colonised. Clinical response to treatment was classified as successful in patients whose infectiondefining signs and symptoms resolved and as failed for patients who deteriorated or whose signs and symptoms persisted.

\section{Statistical analysis of the data}

The data were captured, standardised and analysed using the Statistical Package for Social Sciences (SPSS), version 19 (IBM, USA). The association between underlying conditions and outcome was analysed using Pearson's $\chi^{2}$ test. Logistic regression analysis was used to test for factors associated with survival status of patients.

\section{Results}

\section{Susceptibility of Acinetobacter spp. isolates $(N=107)$}

Six isolates (5.6\%) that were resistant to amikacin were defined as XDR based on their antibiograms. Eighty isolates (80/107, 74.8\%) were MDR. The rest were resistant to fewer than three different classes of tested agents and were therefore not classified as MDR (Table 1). Table 2 shows the antimicrobial MICs of 60 Acinetobacter isolates.

Table 1. Drug resistance patterns of Acinetobacter spp. isolates from clinical specimens $(N=107)$

\begin{tabular}{ll}
\hline Antibiotic susceptibility & $\boldsymbol{n}(\%)$ \\
\hline MDR Acinetobacter spp. & $80(74.8)$ \\
XDR Acinetobacter spp. & $6(5.6)$ \\
PDR Acinetobacter spp. & 0 \\
Amikacin resistant & \\
Resistant to $<3$ tested agents (not MDR) & $6(5.6)$ \\
Total & $15(14.0)$
\end{tabular}

$\mathrm{MDR}=$ multidrug resistant; $\mathrm{XDR}=$ extensively drug resistant; $\mathrm{PDR}$ = pandrug resistant. *Same Acinetobacter spp.

Table 2. $\mathrm{MIC}_{50}$ and $\mathrm{MIC}_{90}$ of the Acinetobacter spp. isolates from clinical specimens $(\mathrm{N}=60)$

\begin{tabular}{|c|c|c|c|c|}
\hline \multirow[b]{2}{*}{ Antibiotics } & \multirow[b]{2}{*}{$\mathrm{MIC}_{50},(\mu \mathrm{g} / \mathrm{mL})$} & \multirow[b]{2}{*}{$\mathrm{MIC}_{90},(\mu \mathrm{g} / \mathrm{mL})$} & \multicolumn{2}{|c|}{ MICs (CLSI) $(\mu \mathrm{g} / \mathrm{mL})$} \\
\hline & & & Sensitive & Resistant \\
\hline CST & 0.25 & 0.5 & $<0.5$ & $>0.5$ \\
\hline IMP & 24 & $>32$ & $<1$ & $>4$ \\
\hline MEM & 24 & $>32$ & $<1$ & $>4$ \\
\hline TZP & $>256$ & $>256$ & 16 & $>32$ \\
\hline $\mathrm{AK}$ & 8 & 16 & 16 & $>64$ \\
\hline CIP & $>32$ & $>32$ & 0.5 & 4 \\
\hline CAZ & $>16$ & $>16$ & 16 & $>16$ \\
\hline
\end{tabular}


Among the 6 amikacin-resistant isolates, the MICs of amikacin ranged between 32 and $\geq 64 \mu \mathrm{g} / \mathrm{mL}$ (Table 2).

\section{Detection of $a p h \mathrm{~A} 6$ and $a a c \mathrm{~A} 4$ genes} Six cases with amikacin-resistant Acinetobacter spp. were identified. The clinical characteristics and outcome of those 6 patients and MICs of tested antibiotics $(n=6)$ are shown in Table 3.

PCR amplification allowed for detection of the aphA6 gene (797 base pair (bp)) from the 6 amikacin-resistant Acinetobacter spp. clinical isolates (Fig. 1). However, the aacA4 gene (489 bp) was not present in these isolates (Fig. 2.).

\section{Phenotypic and genotypic} analysis of the amikacin-resistant Acinetobacter spp.: Correlation of antibiogram with $a p h \mathrm{~A} 6$ and aacA4 genes

The MICs of amikacin and other tested drugs are shown in Table 2. The 6 amikacinresistant strains were sensitive only to colistin and therefore defined as XDR Acinetobacter spp. (Table 3). These 6 strains were phenotypically resistant and showed the presence of the aphA6 gene but not the aacA4 gene (Figs 1 and 2).

\section{Demographic features, clinical} characteristics and outcomes of all patients with infections due to Acinetobacter spp. $(N=107)$

Clinical data were analysed using simple descriptive analysis. The demographic data on patients with Acinetobacter infection $(N=107)$ are shown in Table 4. Acinetobacter spp. were most commonly isolated from adult patients in non-ICU wards and in neonates or paediatric patients.

\section{Underlying diseases}

Acinetobacter spp. were cultured most commonly in adults presenting with trauma and injury, and in paediatric patients with congenitally abnormal organs. Trauma was predominant overall. Retroviral disease, cancer and other conditions showed little risk of colonisation and infection (Table 4). No statistically significant differences $(p>0.05$ $(0.151))$ were observed between children and adults with medical and surgical conditions with regard to the presence of Acinetobacter infections.

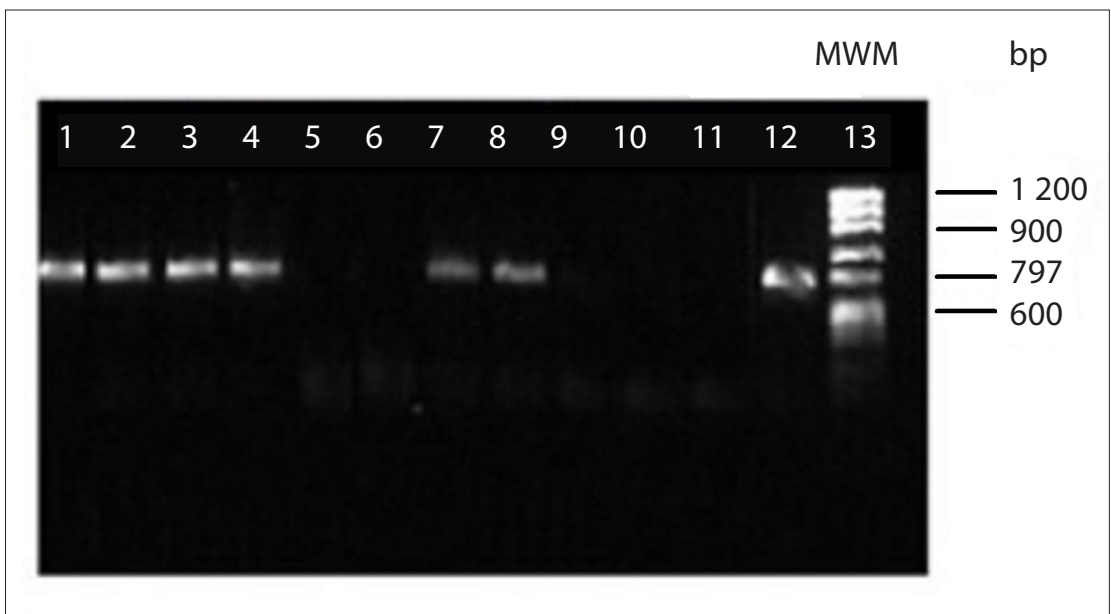

Fig. 1. Polymerase chain reaction for detection of the amikacin-resistant aphA6 gene of Acinetobacter spp. Lanes 1 - 4 and 7 - 8: amikacin-resistant strains (9, 11, 15, 31, 42, 51) (aphA6 gene detected); lanes 5, 6, 9, 10: amikacin-sensitive strains (8, 20, 25, 60) (aphA6 gene bands absent); lane 11: negative control; lane 12: positive control; lane 13: $M W M$. (MWM= molecular weight marker; $b p=$ base pair.)

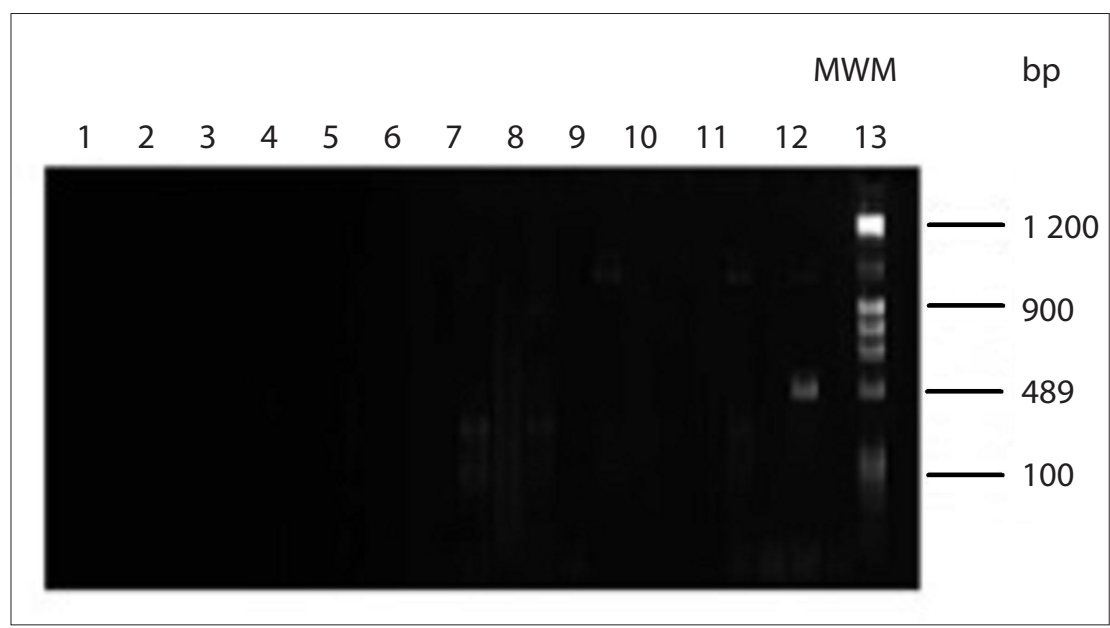

Fig. 2. Polymerase chain reaction for detection of the amikacin-resistant aacA4 gene of Acinetobacter spp. Gene absent in all tested isolates. Lanes 1-11: isolates; lane 12: positive control; lane 13: MWM. ( $M W M=$ molecular weight marker; $b p=$ base pair. $)$

Table 3. Patients' clinical characteristics and outcome, and MICs of other antimicrobial agents tested against amikacin-resistant Acinetobacter spp. $(\mathrm{N}=6)$

\begin{tabular}{|c|c|c|c|c|c|c|c|c|c|c|c|c|}
\hline \multirow[b]{2}{*}{ Isolates } & \multicolumn{7}{|c|}{ MIC $(\mu \mathrm{g} / \mathrm{mL})$} & \multirow[b]{2}{*}{ Ward } & \multirow[b]{2}{*}{ Specimen } & \multicolumn{2}{|l|}{ Days in } & \multirow[b]{2}{*}{ Outcome } \\
\hline & IMP & MEM & AK & TZP & CAZ & CIP & CST & & & hospital & Treated with & \\
\hline AK-R & $>16$ & $>16$ & $>64$ & $>128$ & 64 & $>4$ & $<0.5$ & LW & $\mathrm{BC}$ & 15 & $\begin{array}{l}\mathrm{TZP}+\mathrm{AK} / \mathrm{MEM}+ \\
\mathrm{CST}\end{array}$ & Discharged \\
\hline AK-R & $>16$ & $>16$ & $>64$ & $>128$ & 64 & $>4$ & $<0.5$ & ICU & Pus & 23 & $\mathrm{TZP}+\mathrm{AK}$ & Died \\
\hline AK-R & $>16$ & $>16$ & $>64$ & $>128$ & 64 & $>4$ & $<0.5$ & ICU & ETA & 28 & CST & Discharged \\
\hline AK-R & $>16$ & $>16$ & 32 & $>128$ & 64 & $>4$ & $<0.5$ & $\mathrm{HCU}$ & $\mathrm{BC}$ & 35 & CST & Discharged \\
\hline AK-R & 8 & $>16$ & 32 & $>128$ & 64 & $>4$ & 1 & PU & Pus & 43 & None & Discharged \\
\hline AK-R & $>16$ & $>16$ & 32 & $>128$ & 64 & $>4$ & 0.5 & VU & Pus & 29 & None & Discharged \\
\hline
\end{tabular}




\begin{tabular}{|c|c|c|c|c|}
\hline & $<1$ year $(N=20), n$ & Paediatric $>1$ year $(N=8), n$ & Adult $(N=79), n$ & $p$-value \\
\hline \multicolumn{5}{|l|}{ Sex } \\
\hline Male & 12 & 6 & 46 & \\
\hline Female & 5 & 1 & 31 & \\
\hline NA & 3 & 1 & 2 & \\
\hline \multicolumn{5}{|l|}{ Ward } \\
\hline ICU, paediatric & 5 & 1 & - & \\
\hline Paediatric surgery & 1 & 1 & - & \\
\hline Neonatal & 14 & - & - & \\
\hline Paediatric oncology & - & 2 & - & \\
\hline Paediatric medical unit & - & 1 & - & \\
\hline Trauma & - & 2 & - & \\
\hline NA & - & 1 & 6 & \\
\hline ICU, adult & - & - & 18 & \\
\hline Non-ICU & - & - & 55 & \\
\hline Underlying disease & & & & $0.151(>0.05)$ \\
\hline RVD & 5 & - & 7 & \\
\hline Abnormal organ (congenital) & 10 & - & - & \\
\hline Respiratory disease & 2 & 1 & - & \\
\hline Sepsis & 3 & - & - & \\
\hline Cancer & - & 2 & 3 & \\
\hline Surgical & - & & 17 & \\
\hline Medical & - & 2 & 20 & \\
\hline Injury/trauma & - & 3 & 32 & \\
\hline Antibiotic history & & & & $0.018(<0.05)$ \\
\hline CZT & 1 & & 11 & \\
\hline CZT + combination & - & & 1 & \\
\hline AK (nebulisation) & 1 & 2 & 11 & \\
\hline Others (TZP, CIP, MEM) & 17 & 4 & 30 & \\
\hline No antibiotics given & 1 & 2 & 26 & \\
\hline Outcome & & & & $0.942(>0.05)$ \\
\hline Discharged $(67 / 107,62.6 \%)$ & 10 & 8 & 49 & \\
\hline Died $(23 / 107,21.5 \%)$ & 6 & - & 17 & \\
\hline NA $(17 / 107,15.9 \%)$ & 4 & - & 13 & \\
\hline
\end{tabular}

\section{Antibiotic use}

Tazocin (piperacillin-tazobactam), ciprofloxacin and meropenem were used in most cases. Colistin monotherapy and colistin combinations were not commonly used. Analysis revealed that infections with Acinetobacter spp. were treated mostly with a piperacillin-tazobactam and amikacin combination, while for XDR strains colistin monotherapy or other combinations were used according to the specific characteristics of individual cases (Table 4). Use of colistin, combinations and amikacin differed significantly between adult and paediatric patients $(p<0.05(0.018))$.

\section{Clinical outcome}

The majority of the patients $(67 / 107,62.6 \%)$ were discharged, but mortality was high at $21.5 \%(n=23)$ (Table 4$)$. Clinical outcome was not significantly associated with age $(p>0.05(0.942))$.

Clinical characteristics and outcomes of patients infected with amikacin-resistant Acinetobacter spp. $(n=6)$

All 6 patients with amikacin-resistant Acinetobacter infections were hospitalised in different units for $>2$ weeks ( 21 - 43 days) with chronic illness (Table 3). Two isolates were obtained from blood culture, 3 from pus swabs and 1 from an endotracheal aspirate. Two patients with significant Acinetobacter infections were treated with colistin, while 2 with colonisation received no antibiotics. One of the 6 died, and 5 recovered and were discharged (Table 4).

\section{Discussion}

Despite Acinetobacter spp. being classified by the Infectious Diseases Society of America a decade ago as one of the six most important MDR micro-organisms in hospitals worldwide, ${ }^{[3,4,19]}$ drug-resistant Acinetobacter spp. still present a serious therapeutic and infection control challenge. Increasing antimicrobial resistance among Acinetobacter, resulting in the evolution of XDR and PDR strains, has been documented globally. ${ }^{[12]}$

The present study revealed the presence of amikacin-resistant Acinetobacter spp. at IALCH, with $6(5.6 \%)$ of 107 isolates being amikacin resistant and sensitive only to colistin, defined as XDR Acinetobacter spp.

In our setting, amikacin is commonly used with piperacillintazobactam as a second-line treatment option and amikacin nebulisation for pneumonia cases as general antibiotic policy. Fortunately, 101 (94.4\%) of 107 Acinetobacter spp. isolates were 
highly sensitive to amikacin. In the past, aminoglycosides have played a crucial role in the treatment of infections with MDR Acinetobacter spp. However, Lee et al. ${ }^{[20]}$ reported that Acinetobacter were developing resistance to aminoglycosides around the globe. The current study showed that amikacin-resistant Acinetobacter isolates at IALCH carried the aphA6 gene but not the aacA4 gene. At $5.6 \%$, the prevalence was significantly lower in our local setting than in Korea, according to the 2009 Korean Nationwide Surveillance on Antimicrobial Resistance (KONSAR) study, where amikacinresistant Acinetobacter spp. increased to $48 \% .{ }^{[20]}$

Our data analysis identified a potential emerging challenge to treatment and clinical management that was elucidated by phenotypic and genotypic characterisation of Acinetobacter spp. The study highlights the crucial role of standard amikacin use, as can be seen by the $\mathrm{MIC}_{50}$ and $\mathrm{MIC}_{90}$ of amikacin within the sensitive range, while the $\mathrm{MIC}_{50}$ and $\mathrm{MIC}_{90}$ of imipenem, ciprofloxacin, ceftazidime and piperacillin-tazobactam in the tested isolates were within the highly resistant range (Table 2).

Treatment of MDR Acinetobacter spp. infection usually requires the use of appropriate drugs such as piperacillin-tazobactam plus amikacin, ciprofloxacin, ceftazidime, carbapenem, colistin and tigecycline based on the local antibiogram or individualised microbiological results. Infections with Acinetobacter spp. were mostly treated with piperacillin-tazobactam plus amikacin, whereas colistin monotherapy or combinations were used for XDR Acinetobacter spp. according to the individual case.

Previous studies ${ }^{[1,21,22]}$ have reported MDR Acinetobacter-associated sepsis to be most common in ICU patients. The present study showed that Acinetobacter infections were common in both non-ICU and ICU wards. Infections in the ICU were mainly associated with trauma cases. All isolates were cultured from the specimens after 21 - 43 days of hospitalisation and prior to amikacin exposure.

Infection with Acinetobacter spp. was most prevalent in patients aged 25 - 60 years, and in non-ICU, trauma and postoperative paediatric units. Trauma cases were predominant overall, because Acinetobacter spp. are part of the skin flora and an environmentally acquired organism. Moreover, in the present study, patients in the academic hospital with retroviral disease, cancer and other clinical conditions were not prone to colonisation and infection, possibly because of strict infection prevention and control measures in all high-care units.

The majority of the 107 patients were treated with antibiotics such as piperacillin-tazobactam, amikacin, ciprofloxacin and meropenem according to the local protocol. However, colistin monotherapy, drug combinations and the combination of amikacin with tazocin were used significantly more often in adult patients than paediatric patients $(p<0.05(0.018))$. Infection with XDR Acinetobacter spp. was treated with colistin monotherapy or combinations according to the individual case based on consultation between the clinician and the microbiologist. Our study highlighted that colistin is a key therapeutic option for the treatment of infections with XDR Acinetobacter spp. This finding also indicates the need to enhance infection prevention and control measures and antibiotic stewardship programmes.

As far as we are aware, this study is the first to describe detailed clinical and molecular characteristics of amikacin-resistant Acinetobacter spp. at IALCH, a public academic hospital in KwaZuluNatal. Molecular analysis suggested a potential mechanism of amikacin resistance to be the presence of the aphA6 gene.

Underlying clinical diseases were not significantly associated with clinical outcome in patients with Acinetobacter spp. infections.
A surveillance report for 2016 from the SA private sector ${ }^{[23]}$ showed that $47 \%$ and $37 \%$ of $A$. baumannii isolates were non-susceptible to the aminoglycosides gentamicin and amikacin, respectively. Although the proportion resistant to amikacin has increased in private hospitals, the above study did not include molecular analysis. Molecular epidemiological studies are required when investigating transmission dynamics, which will in turn inform intervention strategies to prevent the spread of drug-resistant strains. Infection prevention and control should also aim to identify reservoirs and sources of infection to recognise and prevent further spread of MDR, XDR and PDR Acinetobacter spp.

\section{Declaration. None.}

Acknowledgements. The authors express their gratitude to Fikile Nkwanyana for assistance with statistical analysis, the medical technologists and clinicians at IALCH who assisted with identification and susceptibility testing and clinical information, and Koleka P Mlisana for general support of the project.

Author contributions. KSS-H, first author and corresponding author: overall writing, formatting the manuscript, literature review, BREC application, co-ordinating the data and input of all contributors; conceptualisation and design of the study, ordering of the laboratory reagents for identification, susceptibility testing and molecular work; as a pathologist (microbiologist), interpretation of laboratory results and regular ward rounds; based on collaboration with clinicians and clinical characterisation and laboratory results of patients, selecting and storing the isolates of Acinetobacter spp. for the study; subculture of the isolates, identification, confirmation of susceptibility tests. MP, second author: contributed to the molecular methods section and molecular work such as DNA extraction; PCR and sequencing, contributed the methodology of the in-house methods; reviewed the methods section of the manuscript. MP, senior author: reviewed and edited the entire manuscript; provided scientific input into the PCR; critically reviewed the drafts and final revised manuscript. All authors read and approved the final manuscript. Funding. The study was funded by the Medical Education Partnership Initiative (MEPI), University of KwaZulu-Natal.

Conflicts of interest. None.

1. Almasaudi SB. Acinetobacter spp. as nosocomial pathogens: Epidemiology and resistance features. Saudi J Biol Sci 2018;25(3):586-596. https://doi.org/10.1016/j.sjbs.2016.02.009

2. Fournier PE, Vallenet D, Barbe V, et al. Comparative genomics of multidrug resistance in Acinetobacter baumannii. PLoS Genet 2006;2(1):e7. https://doi.org/10.1371/journal.pgen.0020007

3. Rice LB. Federal funding for the study of antimicrobial resistance in nosocomial pathogens No ESKAPE. J Infect Dis 2008;197(8):1079-1081. https://doi.org/10.1086/533452

4. Boucher HW, Talbot GH, Bradley JS, et al. Bad bugs, no drugs: No ESKAPE! An update from the Infectious Diseases Society of America. Clin Infect Dis 2009;48(1):1-12. https://doi.org/10.1086/59501 Zhao SY, Jiang DY, Xu PC, et al. An investigation of drug-resistant Acinetobacter baumannii infection in a comprehensive hospital of East China. Ann Clin Microbiol Antimicrob 2015;14(1):7. https://doi. org/10.1186/s12941-015-0066-4

6. Turkoglu M, Mirza E, Tunçcan OG, et al. Acinetobacter species infection in patients with hematologic malignancies in an intensive care unit: Risk factors and impact on mortality. J Crit Care 2011;26(5):460467. https://doi.org/10.1016/j.jcrc.2011.04.007

7. El-Mahallawy H, Sidhom I, El-Din NHA, Zamzam M, El-Lamie MM. Clinical and microbiologic determinants of serious bloodstream infections in Egyptian pediatric cancer patients: A one-year study. Int J Infect Dis 2005;9(1):43-51. https://doi.org/10.1016/j.ijid.2003.11.010

8. Sievert DM, Ricks P, Edwards JR, et al. Antimicrobial-resistant pathogens associated with healthcareassociated infections summary of data reported to the National Healthcare Safety Network at the Centers for Disease Control and Prevention, 2009 - 2010. Infect Control Hosp Epidemiol 2013;34(1):14. https://doi.org/10.1086/668770

9. Magiorakos AP, Srinivasan A, Carey RB, et al. Multidrug-resistant, extensively drug-resistant and pandrug-resistant bacteria: An international expert proposal for interim standard definitions for acquired resistance. Clin Microbiol Infect 2012;18(3):268-281. https://doi.org/10.1111/j.14690691.2011.03570.x

10. Peleg AY, Seifert H, Paterson DL. Acinetobacter species: Emergence of a successful pathogen. Clin Microbiol Rev 2008;21(3):538-582. https://doi.org/10.1128/CMR.00058-07

11. Munoz-Price LS, Arheart K, Nordmann P, et al. Eighteen years of experience with Acinetobacter species in a tertiary care hospital. Crit Care Med 2013;41(12):2733-2742. https://doi.org/10.1097/
CCM.0b013e318298a541

12. Manchanda V, Sanchaita S, Singh NP. Multidrug resistant Acinetobacter. J Glob Infect Dis 2010;2(3):291-304. https://doi.org/10.4103/0974-777X.68538 
13. Nwadike VU, Ojide CK, Kalu EI. Multidrug resistant Acinetobacter infection and their antimicrobial susceptibility pattern in a Nigerian tertiary hospital ICU. Afr J Infect Dis 2014;8(1):14-18. https://doi. org/10.4314/ajid.v8i1.4

14. Aliakbarzade K, Farajnia S, Karimi Nik A, Zarei F, Tanomand A. Prevalence of aminoglycoside resistance genes in Acinetobacter baumannii isolates. Jundishapur J Microbiol 2014;7(10):e11924. resistance genes in Acinetobacter
https://doi.org/10.5812/jim.11924

15. Shaw KJ, Rather PN, Hare RS, Miller GH. Molecular genetics of aminoglycoside resistance genes and familial relationships of the aminoglycoside-modifying enzymes. Microbiol Rev 1993;57(1):138-163. familial relationships of the aminog/ycos

16. Miller GH, Sabatelli FJ, Naples L, Hare RS, Shaw KJ. The most frequently occurring aminoglycoside Miller GH, Sabatelli FJ, Naples L, Hare RS, Shaw KJ. The most frequently occurring aminoglycoside
resistance mechanisms - combined results of surveys in eight regions of the world. The Aminoglycoside resistance mechanisms - combined results of surveys in eight reg
Resistance Study Groups. J Chemother 1995; 7 (Suppl 2):17-30,

Resistance Study Groups. J Chemother 1995; (Suppl 2):17-30. 7. Clinical and Laboratory Standards Institute. Performance Standards for Antim
Testing: 24th Informational Supplement; M100-S24. Wayne, Penn.: CLSI, 2014.

18. Swe Swe-Han K, Pillay M, Schnugh D, Mlisana KP, Baba K, Pillay M. Horizontal transfer of OXASwe Swe-Han K, Pillay M, Schnugh D, Mlisana KP, Baba K, Pillay M. Horizontal transfer of OXA-
23-carbapenemase-producing Acinetobacter species in intensive care units at an academic complex hospital, Durban, KwaZulu-Natal, South Africa. South Afr J Infect Dis 2017;1(1):1-8. https://doi.org/ $10.1080 / 23120053.2017 .1335482$
19. Qureshi ZA, Hittle LE, O'Hara JA, et al. Colistin-resistant Acinetobacter baumannii: Beyond carbapenem resistance. Clin Infect Dis 2015;60(9):1295-1303. https://doi.org/10.1093/cid/civ048

20. Lee K, Yong D, Jeong SH, Chong Y. Multidrug-resistant Acinetobacter spp.: Increasingly problematic nosocomial pathogens. Yonsei Med J 2011;52(6):879-891. https://doi.org/10.3349/ymj.2011.52.6.879

21. Uwingabiye J, Frikh M, Lemnouer A, et al. Acinetobacter infections prevalence and frequency of the antibiotics resistance: A comparative study of intensive care units versus other hospital units. Pan Afr Med J 2016;23(1):191. https://doi.org/10.11604/pamj.2016.23.191.7915

22. Swe Swe-Han K, Pillay M. Multidrug resistant Acinetobacter species: A significant cause of sepsis in an intensive care unit in a regional hospital, Durban. Int J Nurs Didactics 2015;5(8). www. innovativejournal.net/index.php/ijnd/article/download/1745/1397 (accessed 2 December 2019).

23. Perovic $\mathrm{O}$, Ismail $\mathrm{H}$, van Schalkwyk $\mathrm{E}$, et al. Antimicrobial resistance surveillance in the South African private sector report for 2016. South Afr J Infect Dis 2018;33(4):114-117. https://doi.org/10.1080/231 20053.2018.1482646

Accepted 16 September 2019 